\title{
Examining the Relationship between Trait Self-control and Stress Evidence on Generalizability and Outcome Variability
}

\author{
Nielsen, Kristian S.; Bauer, Jan Michael; Hofmann, Wilhelm
}

Document Version

Accepted author manuscript

Published in:

Journal of Research in Personality

DOI:

10.1016/j.jp.2019.103901

Publication date:

2020

\section{License \\ CC BY-NC-ND}

Citation for published version (APA):

Nielsen, K. S., Bauer, J. M., \& Hofmann, W. (2020). Examining the Relationship between Trait Self-control and Stress: Evidence on Generalizability and Outcome Variability. Journal of Research in Personality, 84, [103901] https://doi.org/10.1016/j.jrp.2019.103901

Link to publication in CBS Research Portal

\section{General rights}

Copyright and moral rights for the publications made accessible in the public portal are retained by the authors and/or other copyright owners and it is a condition of accessing publications that users recognise and abide by the legal requirements associated with these rights.

Take down policy

If you believe that this document breaches copyright please contact us (research.lib@cbs.dk) providing details, and we will remove access to the work immediately and investigate your claim. 


\title{
Examining the Relationship between Trait Self-control and Stress: Evidence on Generalizability and Outcome Variability
}

\author{
Kristian S. Nielsen, Jan Michael Bauer, and Wilhelm Hofmann
}

Journal article (Accepted manuscript*)

\section{Please cite this article as:}

Nielsen, K. S., Bauer, J. M., \& Hofmann, W. (२०२०). Examining the Relationship between Trait Self-control and Stress: Evidence on Generalizability and Outcome Variability. Journal of Research in Personality, 84, [103901]. https://doi.org/10.1016/j.jp.2019.103901

DOl: https://doi.org/10.1016/j.jp.2019.103901

* This version of the article has been accepted for publication and undergone full peer review but has not been through the copyediting, typesetting, pagination and proofreading process, which may lead to differences between this version and the publisher's final version AKA Version of Record.

Uploaded to CBS Research Portal: March २०२1

(C) 2019. This manuscript version is made available under the CC-BY-NC-ND 4.0 license http://creativecommons.org/licenses/by-nc-nd/4.0/ 


\title{
Examining the Relationship Between Trait Self-Control and Stress: Evidence on Generalizability and Outcome Variability
}

\author{
Kristian S. Nielsen \\ University of Cambridge \\ Jan M. Bauer \\ Copenhagen Business School \\ Wilhelm Hofmann \\ Ruhr-University Bochum
}

This manuscript has been accepted for publication in Journal of Research in Personality. Please note that some changes may occur during the copy-editing process, such that the final published version may differ somewhat from this version.

Please address correspondence concerning this article to:

Kristian Steensen Nielsen

Department of Zoology

University of Cambridge

Pembroke Street

CB2 3EJ Cambridge

United Kingdom

ksn27@cam.ac.uk 


\begin{abstract}
Trait self-control has in several studies been found negatively linked to stress. These studies have, however, mostly relied on student and/or one-country samples. Study 1 investigated the generalizability of the relationship between trait self-control and stress through a four-country survey $(\mathrm{N}=4,097)$. The results showed consistently strong and negative relationships between trait self-control and stress across the four countries. Study 2 investigated the relationship between trait self-control and daily stress through a two-week diary study $\left(\mathrm{N}=594 ; \mathrm{n}_{\mathrm{obs}}=7,880\right)$, showing that trait self-control was negatively related to daily stress and stress variability. Together, the two studies show that trait self-control's negative link to stress generalizes beyond students and the United States.
\end{abstract}

Keywords: trait self-control, stress, mental health, cross-country survey, diary study 


\section{Introduction}

Self-control has traditionally been conceptualized as the ability to override or change one's inner responses, as well as to interrupt behavioral tendencies, such as impulses, and refrain from acting on them (Tangney, Baumeister, \& Boone, 2004; but see Milyavskaya, Berkman, \& de Ridder, 2019). Much research has investigated the correlates of adept self-control at both the state and trait level. Especially trait self-control has been found positively associated with a wealth of positive and desired life outcomes (De Ridder, Kroese, \& Gillebaart, 2018). These outcomes include stronger interpersonal relationships, better academic performance, and higher levels of subjective well-being (De Ridder, Lensvelt-Mulders, Finkenauer, Stok, \& Baumeister, 2012; Galla \& Duckworth, 2015; Hofmann, Luhmann, Fisher, Vohs, \& Baumeister, 2014; Nielsen, Gwozdz, \& de Ridder, 2019). Another, often-reported benefit of high trait self-control is better mental health, as indicated by better psychological adjustment and lower levels of stress, anxiety, and depression. The link to mental health was first reported by Tangney et al. (2004) who found that trait self-control was negatively correlated with indicators of poor psychological adjustment including depression and anxiety. Subsequent studies have supported the negative relations between trait self-control and stress, anxiety, and depression (Baldwin, Garrison, Crowell, \& Schmeichel, 2018; Boals, vanDellen, \& Banks, 2011; Bowlin \& Baer, 2012; Hofmann et al., 2014).

In the present investigation, we particularly focus on trait self-control's link to one aspect of mental health, namely that of stress. Trait self-control may influence stress in a number of ways. For example, people with high trait self-control often ameliorate stressful situations more competently by employing effective regulation strategies (Hennecke, Czikmantori, \& Brandstätter, 20189; Hofmann, Baumeister, Förster, \& Vohs, 2012) and selecting themselves into more supportive social environments (Nielsen \& Bauer, 2019; vanDellen, Shah, Leander, Delose, \& Bornstein, 2015). Moreover, people with low trait self-control may experience greater and more frequent problems with regulating thoughts and behavior (De Ridder et al., 2012). 
Stress can equally influence both the development of trait self-control and momentarily reduce people's ability to exert self-control. For example, childhood stress can negatively impact brain and cognitive development; specifically, it has been linked to reduced neural activity in the prefrontal cortex and poorer executive functions in adults (Blair, 2010; Hackman \& Farah, 2009; Sapolsky, 1994). Experiencing stress as an adult can also temporarily impede executive functions (Arnsten, 2009; Blair \& Ursache, 2011; Starcke \& Brand, 2012), making effective self-control even harder (Hofmann, Schmeichel, \& Baddeley, 2012).

Given these different dynamics dovetailing a negative link between trait self-control and stress, one would expect a strong empirical base supporting this relationship. However, the evidence substantiating this relationship does not appear to be particularly strong with most studies being based on smaller student samples, typically from the United States (Baldwin et al., 2018; Boals et al., 2011; Bowlin \& Baer, 2012; Hisler, Krizan, \& DeHart, 2018; Hofmann et al., 2014). To the best of our knowledge, whether the negative relationship between trait self-control and stress generalizes to other population segments and cross-culturally thus remains unknown.

In two high-powered studies, we investigated the relationship between trait self-control and stress. Study 1 assessed the correlation between trait self-control and self-reported stress through a large four-country survey. This study also assessed self-reported anxiety and depression to investigate trait self-control's association to other mental health indicators. Study 2 addressed the shortcomings of Study 1's cross-sectional design by examining the relationship between trait selfcontrol and daily stress through a large two-week diary study conducted in the United Kingdom ${ }^{1}$.

\footnotetext{
${ }^{1}$ The two studies' analyses were not preregistered; however, Study 2's research protocol was preregistered (osf.io/ut6kp). The data cannot be shared due to the funding projects' data publication policies. The first author led the research conceptualization with inputs from the second and third author. The first author collected the data, and the second author conducted the data analyses. The first and second author wrote the manuscript with substantial edits from the third author.
} 


\section{Study 1: Cross-country analysis}

Study 1 was motivated by the (re-)emerging concerns raised about the generalizability of psychological research (Henrich, Heine, \& Norenzayan, 2010; Rad, Martingano, \& Ginges, 2018). The generalizability concerns, amongst other, refer to the prevalent reliance on undergraduate student samples and online convenience samples from the United Kingdom and United States, and the typically limited justification for how and why psychological findings are expected to generalize to a wider population (Arnett, 2008; Rad et al., 2018; Simons, Shoda, \& Lindsay, 2017). Study 1 aimed to address these concerns by investigating the generalizability of trait self-control's negative relationship with stress through surveying socio-demographically diverse samples in Germany, Poland, Sweden, and United States. In each country, we assessed self-reported stress, as well as anxiety and depression representing other indicators of mental health. These measures allowed us to assess whether trait self-control relates equally to different aspects of mental health.

\subsection{Method}

An extensive survey, administered by Qualtrics in the period between October 2016 and January 2017, was distributed in four countries: Germany, Poland, Sweden, and the United States. The survey was developed to assess environmental aspects of clothing consumption, but also included measures of trait self-control and mental health indicators. Because the survey was not solely developed for the present purpose, we note that other studies have been published based on the same dataset (Gwozdz, Nielsen, \& Müller, 2017; Joanes, 2019; Nielsen \& Bauer, 2019; Nielsen, Gwozdz, \& de Ridder, 2019). The survey was originally developed in English and then translated into German, Polish, and Swedish by qualified translators certified with ISO17100. Participants were incentivized for their participation in the form of points redeemable for various products (e.g., gift cards). Due to its comprehensiveness, the survey was divided into two parts that were completed within a two- to four-week interval (all variables discussed here were included in Part II 
except for demographics). Only respondents completing both survey parts were included in the final sample. Part I was representative of age, gender, region, and education. But as participants could decide whether or not to participate in Part II - subjecting the process to self-selection bias - full representativeness was not achieved. This led to especially younger, low-educated men being under-sampled.

The total sample consisted of 4,591 respondents $(\mathrm{N}=10,363$ completed Part I). However, 514 respondents were excluded from the analysis due to incomplete responses (i.e., missing answers for all items in one or more scales; descriptive statistics of excluded cases are available upon request). This resulted in a final sample of 4,097 with the following breakout by country: Germany $(n=$ $1,080)$, Poland $(n=978)$, Sweden $(n=1,047)$, and the United States $(n=992)$. The mean age of the final sample was $42.59(S D=13.47)$ with $56.6 \%$ being female.

\subsubsection{Materials}

Trait self-control was measured through the well-validated Brief Self-Control Scale (Tangney et al., 2004) consisting of 13 items. Participants answered on a 7 -point likert scale $(1=$ not at all to $7=$ very mисh) to indicate their general self-control tendencies. Example items are: "I am good at resisting temptation," "People would say that I have iron self-discipline," and "I am able to work effectively toward long-term goals.” The overall Cronbrach alpha was .85 with little deviations on the country level ${ }^{2}$. For our analytical approach, we followed the recommendation of Enders and Tofighi (2007) and created z-scores based on the individual country distributions.

Stress, anxiety, and depression were measured through the Depression Anxiety Stress Scale (DASS-21; Lovibond \& Lovibond, 1995). The scale consists of three dimensions containing a total of 21 items measuring negative affect and bodily symptoms. The items were rated by participants

\footnotetext{
${ }^{2}$ Country-specific alphas: Germany $(\alpha=.86)$; Poland $(\alpha=.83)$; Sweden $(\alpha=.83)$; United States $(\alpha=.87)$.
} 
on a severity/frequency scale from 0 (did not apply to me) to 3 (applied to me very much or most of the time) relating to their experiences over the past week. Scores for stress, anxiety, and depression were calculated by summing the scores for the relevant items with a maximum value of 21 per dimension. The summed scores were then multiplied by two to make them comparable with the full DASS-42 scale and its severity ratings. Each dimension could therefore range between 0 and 42 .

The alphas for the instruments of the total sample were: stress $(\alpha=.88)$, anxiety $(\alpha=.86)$, and depression $(\alpha=.93)$ and without any noteworthy differences on the country level. We leave these uncentered for our main analysis (resulting in semi-standardized regression coefficients). Control variables were age (measured in years) and sex.

\subsection{Results}

Descriptive data showed that the average reported levels of stress $(M=11.95, S D=9.94)$, anxiety $(M=7.16, S D=8.68)$, and depression $(M=10.47, S D=10.95)$ were generally classified as 'normal' across all countries, according to the ratings by Lovibond \& Lovibond (1995), except for depression and anxiety levels in Poland and the United States (see Table S1 in the Supplemental Material for country-specific descriptive data). The correlations of the pooled sample in Table 1 reveal two noteworthy patterns. All three outcome variables of the DASS21 were highly correlated and showed a consistently negative relationship with trait self-control.

Table 1. Intercorrelations of pooled sample

\begin{tabular}{lrrrrrrr}
\hline \hline & $M$ & $S D$ & Range & 1 & 2 & 3 & 4 \\
\hline 1. Stress & 11.95 & 9.94 & $0-42$ & - & & \\
2. Anxiety & 7.16 & 8.68 & $0-42$ & $.747^{* * *}$ & - & \\
3. Depression & 10.47 & 10.95 & $0-42$ & $.779^{* * *}$ & $.725^{* * *}$ & - & \\
4. Trait self-control (uncentered) & 4.44 & 1.01 & $1-7$ & $-.410^{* * * *}$ & $-.328^{* * * *}$ & $-.397^{* * *}$ & - \\
\hline Note. Correlations significant at ${ }^{*} p<.05,{ }^{* * *} p<.01,{ }^{* * * *} p<.001$. & & & & &
\end{tabular}


For our main analysis of the relationship between trait self-control and the mental health indicators, three OLS regressions with stress, anxiety, and depression as dependent variables were run on the pooled data and for each country. The analyses included age and sex as co-variates. Table 2 displays the regression results. For stress, trait self-control exhibited a negative, statistically significant relationship. This effect was observed for the pooled sample but also for each country individually. A similar pattern was observed for pooled sample of anxiety and depression, as well as for the individual country results (see Table 2).

Table 2. Relationship between trait self-control and mental health indicators

\begin{tabular}{|c|c|c|c|c|c|}
\hline & Full & Germany & Poland & Sweden & United States \\
\hline \multicolumn{6}{|l|}{$D V:$ Stress } \\
\hline $\begin{array}{l}\text { Trait self-control } \\
\text { (z-score) }\end{array}$ & $\begin{array}{c}-3.51^{* * *} \\
{[-3.80,-3.23]}\end{array}$ & $\begin{array}{c}-3.34^{* * * *} \\
{[-3.93,-2.74]}\end{array}$ & $\begin{array}{c}-2.60^{* * *} \\
{[-3.17,-2.02]}\end{array}$ & $\begin{array}{c}-3.02^{* * *} \\
{[-3.52,-2.53]}\end{array}$ & $\begin{array}{c}-5.15^{* * *} \\
{[-5.75,-4.55]}\end{array}$ \\
\hline \multicolumn{6}{|l|}{ DV: Anxiety } \\
\hline $\begin{array}{l}\text { Trait self-control } \\
\text { (z-score) }\end{array}$ & $\begin{array}{c}-2.50^{* * * *} \\
{[-2.78,-2.23]}\end{array}$ & $\begin{array}{c}-2.14^{* * *} \\
{[-2.67,-1.61]}\end{array}$ & $\begin{array}{c}-2.18^{* * *} \\
{[-2.78,-1.58]}\end{array}$ & $\begin{array}{c}-1.92^{* * *} \\
{[-2.37,-1.47]}\end{array}$ & $\begin{array}{c}-3.97^{* * * *} \\
{[-4.58,-3.35]}\end{array}$ \\
\hline \multicolumn{6}{|l|}{ DV: Depression } \\
\hline $\begin{array}{l}\text { Trait self-control } \\
\text { (z-score) }\end{array}$ & $\begin{array}{c}-3.99^{* * *} \\
{[-4.32,-3.67]}\end{array}$ & $\begin{array}{c}-3.69^{* * *} \\
{[-3.97,-2.68]}\end{array}$ & $\begin{array}{c}-3.32^{* * *} \\
{[-3.97,-2.67]}\end{array}$ & $\begin{array}{c}-3.40^{* * *} \\
{[-4.03,-2.77]}\end{array}$ & $\begin{array}{c}-5.56^{* * *} \\
{[-6.31,-5.00]}\end{array}$ \\
\hline
\end{tabular}

Note: Each cell in this table reports the coefficient from regressing stress, anxiety and depression on trait selfcontrol (semi-standardized effects). All regressions used age and sex as co-variates. The three regressions using the full sample, displayed in column 1, also included country dummies. The full regression tables are provided in the Supplemental Material. Heteroscedasticity robust standard errors for the OLS regression in parentheses. ${ }^{*} p<.05,{ }^{* *} p<.01,{ }^{* * *} p<.001$.

Examining the results between countries, the coefficients for Germany, Poland, and Sweden were comparable in magnitude, while the U.S. sample showed an overall stronger relationship. This pattern also became evident when looking at different effect size estimates. For stress, the fully standardized coefficients of trait self-control ranged from -.28 for Poland to -.46 in the U.S. sample. Using partial omega-squares, we found between 8 to $22 \%$ of the variability in the stress (z-score) explained by our main variable of interest. While similar effect sizes were obtained for depression, 
the link between trait self-control and anxiety appeared slightly weaker (for details see Table S2). We also tested whether the country dummies significantly moderated the relationship between trait self-control in the full sample for the three dependent variables. Displayed in Figure 1, we observed that the relationship between trait self-control and the three mental health indicators was stronger in the United States when compared to the three other countries. All country-specific slopes were, however, significantly different from zero and full regression tables are provided in the Supplemental Material (Table S3-S5). With minor exceptions regarding the interaction terms, the observed patters remained robust when using the raw trait self-control score and a negative binomial model to account for the non-normal distribution of the dependent variable (see Table S6S7; Figure S1-S3).
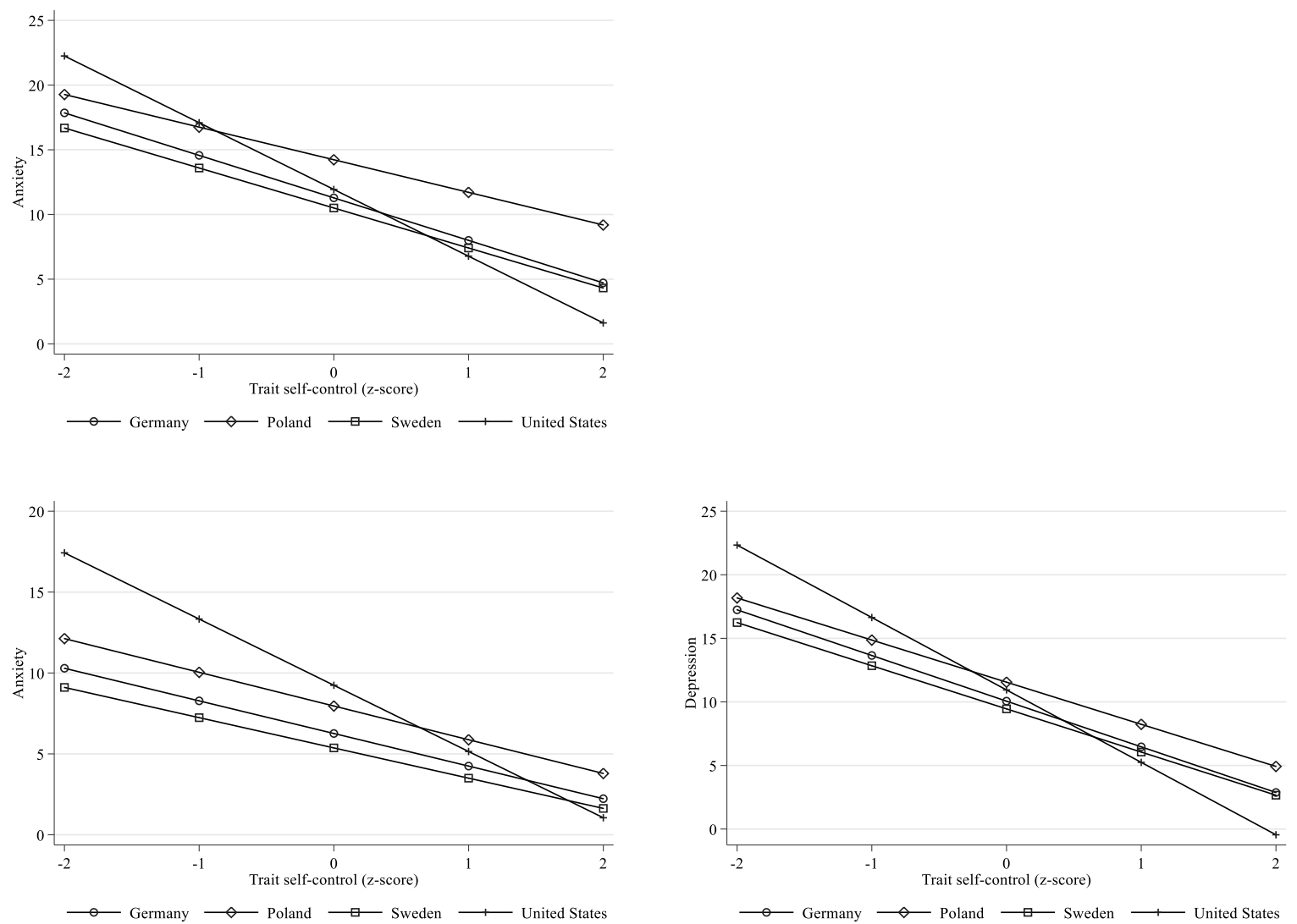

Figure 1. Relationship between trait self-control and mental health by country 


\subsection{Discussion}

The first study investigated the generalizability of the negative relationship between trait selfcontrol and stress. Our analysis, which also included anxiety and depression as other indicators of mental health, supports the generalizability of this relationship to at least Germany, Poland, Sweden, and the United States. Specifically, we showed that trait self-control was associated with lower self-reported levels of stress, anxiety, and depression. This effect was strong and consistent across the four countries. We did observe between-country differences, where mainly the U.S. sample exhibited a comparably stronger relationship between trait self-control and our three mental health measures. We can only speculate on possible reasons for this country difference. These may include the greater income inequality in the United States, affecting both self-control and mental health (Farah, 2017; Moffit et al., 2011; Pickett \& Wilkinson, 2010), and the comparably weaker social-security net, which might shield people against adversities in life linked to their limited selfcontrol ability.

In sum, Study 1 provides cross-cultural evidence for trait self-control's negative relationship with stress, as well as for anxiety and depression. In study 2 , we sought to further advance this evidence by examining the relationship between trait self-control and daily stress through a twoweek diary study. Specifically, we assessed whether trait self-control predicted within-person variability in daily stress.

\section{Study 2: Intense longitudinal evidence on daily stress}

The objective of Study 2 was to provide intense-longitudinal and more ecologically valid evidence for the relationship between trait self-control and stress. Moreover, in the effort of dissecting this relationship, we aimed to advance Study 1 by investigating whether trait self-control would predict within-person variability in daily stress levels. To accomplish these objectives, we turned to a recently conducted diary study on self-control and clothing consumption where participants 
reported their perceived stress level on every day for two full weeks. In line with the results of Study 1 and previous research (Baldwin et al., 2018; Hofmann et al., 2014), we hypothesized that trait self-control would negatively correlate with daily stress. Beyond mean differences, we explored a hypothesis relating to the fluctuation in daily stress levels. Because people with high trait self-control are generally more competent at regulating their thoughts, emotions, and behavior, we hypothesized that trait self-control would be negatively correlated with fluctuations in daily stress levels. The intense-longitudinal data provided by the diary method offered an ideal test of this novel hypothesis.

\subsection{Method}

\subsubsection{Participants}

A two-week diary study was distributed to people residing in the United Kingdom. To help recruit study participants and to facilitate the data collection, we used the online research platform, Prolific. Our objective was to have the highest attainable statistical power; thus, we sampled as many participants as our budget allowed for. This yielded a goal of 700 participants. The recruitment stopped when this target was reached, resulting in an initial sample of 705 participants who all completed the intake study. We instituted a payment eligibility requirement with the aim of securing a high response rate. This meant that participants had to complete a minimum of 11 out of 14 daily protocols in order receive payment. All participants were informed of this eligibility requirement in the study description on Prolific's platform, prior to completing the intake survey, and at the end of every daily diary protocol. The payment eligibility requirement along with participant qualification requirements and the diary protocol were preregistered on OSF (osf.io/ut6kp). A total of 105 participants failed to meet the payment eligibility requirement and were therefore excluded from further analysis. An additional six participants were excluded following technical issues with the daily response reminders. This resulted in a final sample of 594 
adults, comprising $71 \%$ females with an average of $37.44(S D=11.07$; age range: $18-64$ years). The sample was, on average, more educated (31\% with college/A-levels, $34 \%$ with undergraduate degree, $15 \%$ with graduate or doctorate degree) and with more left-leaning political beliefs than the U.K. average (37\% leftwing, 24\% center, $13 \%$ rightwing, and $24 \%$ reported not knowing). The recruited participants differed in their employment status: full-time employed (34\%), part-time employed (24\%), homemakers (15\%), self-employed (11\%), out of work (5\%), students (5\%), retired $(3 \%)$, and unable to work $(3 \%)$.

\subsubsection{Procedure and materials}

Participants completed an intake survey on the $4-5^{\text {th }}$ of December and on the $6^{\text {th }}$ of December the diary study begun with daily reporting over the following 14 days. All participants who met the payment eligibility requirement were compensated with $£ 10$ for their participation —independent of whether they completed 11 or 14 daily protocols. Throughout the assessment period, participants received a link every day at 5:00 pm to the daily survey, which was sent through Prolific's mailing system. The link expired 8:00 am the following day. Across the two weeks, the average completion rate was $82.93 \%$ for all participants invited to the diary study $(\mathrm{N}=705)$ and $96.06 \%$ for the final sample $(\mathrm{N}=594)$. Due to technical issues on Day 4, which resulted in a two-hour delay in the publication of the survey link, the completion rate was considerably lower on this day. The total number of diary responses was 7,880.

The intake survey included measurements of psychological traits, subjective well-being, consumption-related goals, and past clothing consumption. The diary protocol included a wide range of measurements of desire experiences, self-control, and clothing consumption. For the purpose of this study, we focus on the measure of daily stress level that was asked in every protocol: "How stressed did you feel since the last time we asked" (on day 1 the measure read "How stressed did you feel today"). This question was rated on a 7-point likert scale $(1=$ not 
stressed at all, $7=$ very stressed). Trait self-control was measured in the same way as in Study 1 and z-scores were created on the overall distribution (Tangney et al., 2004). All measurements in the intake survey and diary protocol can be found at OSF ( $\underline{\text { osf.io/ut6kp}) . ~}$

\subsubsection{Data analytic strategy}

To assess the effects of trait self-control on daily stress, we employed a similar analytical strategy to Study 1 . However, the diary data had a clear hierarchical structure. Information about sex, age, and personality traits were collected in the intake survey and did not vary over time (Level 2), while the daily stress reports were nested within participants (Level 1). As the individual stress measures were correlated (intracluster correlation coefficient $=.30$ ), we used clustered OLS standard errors on Level 2 to avoid overly narrow confidence intervals (Rogers, 1993). This method has been suggested as a more straightforward and practical approach for data with a comparably simple nested structure (Primo, Jacobsmeier, \& Milyo, 2007). We also supply the results of a multilevel random-effects model for comparison, which provided similar results. As in Study 1, we used age and sex as control variables in all regressions.

\subsection{Results}

Trait self-control consistently predicted lower stress levels over the 14-day period. The results from

OLS, our main method, showed that an increase of one standard deviation in trait self-control was associated with -.22 points of the daily stress measure $(t=-4.80,95 \% \mathrm{CI}=[-.31,-.13], p<.001)$. The similar results from an ordered logit and a multilevel random effects model showed that our findings were not sensitive to a specific modelling approach (see Table S8 for detailed results). These results support our first hypothesis and the findings from Study 1 that trait self-control is negatively related to daily stress. 


\subsubsection{Within-person variability}

We next tested whether trait self-control predicted the within-person variability of daily stress. For this analysis, we collapsed the longitudinal data on the individual level. We calculated the variability of stress by the within-person standard deviation (SD) of the daily stress reports $(\mathrm{N}=$ 594) and obtained the average stress for each individual over the 14-day period. Individual observations used to calculate the SD varied between 11 and 14 daily reports. The descriptive distribution of scores appeared very close to normal (see Figure S4).

The regression results, presented in the first, left column of Table 3, showed that people with high trait self-control had significantly lower average daily stress, thus mimicking the results from the longitudinal data presented in the previous section $(\beta=-.23, t=-4.90,95 \% \mathrm{CI}=[-.31,-.13], p<$ .001). The models in the middle and right column of Table 3 have stress variability as the dependent variable. When estimating this within-person variability in the middle column, we found that trait self-control also predicted a lower variation during the 14 -day period $(\beta=-.06, t=-2,64,95 \% \mathrm{CI}=$ $[-.10,-.01], p=.009)$. Given that stress was reported on a scale from 1 to 7 , we might be concerned about potential ceiling effects. If stress levels are particularly low or high, the measure technically prohibits large fluctuations beyond the endpoints of the scale. We alleviated this potential concern by adding participants' average stress levels as control to the model displayed in the right column of Table 3: the results remained remarkably similar $(\beta=-.05, t=-2.15,95 \% \mathrm{CI}=[-.09,-.01], p=$ $.032)$. 
Table 3. Trait self-control on variability of daily stress

\begin{tabular}{lccc}
\hline & $\begin{array}{c}\text { Average daily } \\
\text { stress }\end{array}$ & Stress variability & $\begin{array}{c}\text { Stress variability controlling } \\
\text { for mean stress }\end{array}$ \\
\hline Trait self-control (z-score) & $-.23^{* * *}$ & $-.06^{* *}$ & $-.05^{*}$ \\
Average daily stress & {$[-.32,-.14]$} & {$[-.10,-.01]$} & {$[-.09,-.00]$} \\
& & 04 \\
Age & -.01 & $-.00^{*}$ & {$[-.01, .08]$} \\
& {$[-.02, .00]$} & $-.01,-.00]$ & $-.00^{*}$ \\
Female & -.05 & $.24^{* * *}$ & {$[-.01,-.00]$} \\
Constant & {$[-.23, .14]$} & {$[-15, .33]$} & $.24^{* * *}$ \\
& $3.74^{* * *}$ & $1.32^{* * *}$ & {$[.16, .33]$} \\
& {$[3.41,4.07]$} & {$[1.16,1.48]$} & {$\left[.18^{* * *}\right.$} \\
$N$ & 594 & 594 & $1.42]$ \\
adj. $R^{2}$ & .06 & .07 & 594 \\
\hline
\end{tabular}

Note. Column 1 present a replication of the results in Table $\mathrm{S} 7$ but uses the overall average rather than nested panel data. The models in column 2 and 3 show the link between trait self-control and the within-person variability of stress (within-subject SD over the whole study period). Controls are the age of the subject, and female displays the effect of a sex dummy indicating 1 if the subject was female. The $95 \%$ confidence intervals are based on heteroskedastic robust standard errors indicated in brackets. ${ }^{*} \mathrm{p}<.05,{ }^{* *} \mathrm{p}<.01,{ }^{* * *} \mathrm{p}<.001$.

\subsection{Discussion}

The second study provides intense-longitudinal support with greater ecological validity for the negative relationship between trait self-control and stress, showing that trait self-control is negatively related to daily stress. The study also showed that trait self-control is negatively associated with stress variability, even when controlling for average stress levels (we unpack this novel finding in the general discussion). Future research might try to replicate and explain this intriguing effect emerging from our exploratory analysis.

\section{General discussion}

Results from two large studies strongly support the generalizability of the negative relation between trait self-control and stress. Study 1 showed that trait self-control was negatively related to stress across large and diverse population segments in Germany, Poland, Sweden, and United States (overall $N=4,097$ ). The study also found similar negative relationships between trait self-control 
and anxiety and depression. These findings represent an important extension of previous investigations, which have predominantly been based on student samples (Boals et al., 2011; Bowlin \& Baer, 2012; Tangney et al., 2004). Study 2 showed that trait self-control negatively predicted daily stress and interestingly also stress variability. This means that people with high trait self-control not only displayed lower average stress levels over the study period, but also showed lower day-to-day fluctuations in their perceived stress. The relationship between trait self-control and stress variability has, to our knowledge, not been investigated before, and thus this study adds a novel perspective to how trait self-control relates to stress.

Our results also have clinical implications. Prolongued stress responses have been linked to poorer mental health outcomes such as depression (e.g., Yang et al., 2015). In fact, some of the symptoms of chronic stress appear to overlap with symptoms of anxiety or depression (e.g., Lovibond \& Lovibond, 1995). Our own data and supplemental analyses clearly support such a connection: First, stress, anxiety, and depression were substantially intercorrelated. Second, supplementary regresssion analyses in Study 1 also established trait self-control as a highly reliable predictor of anxiety and depression as well. Hence, we believe that the present key findings regarding the connection between trait self-control and stress can be generalized to other construct of mental health, defined more broadly. In this sense, the present research provides a bridge between trait self-control, as typically in the focus of motivation and personality science, and clinical psychology.

The potential causal pathways that link trait self-control and stress (mean and variability) are manifold. Higher trait self-control already manifest in childhood and adolescence and can lead to overall better living conditions that reduce the frequency and intensity of stressors (e.g., Mischel, Shoda, \& Rodriguez, 1989; Moffitt et al., 2011). Being linked to better education and carrier outcomes, trait self-control may thus act through the absence of financial concerns and hardship. Differences in socioeconomic circumstances may directly contribute to lower stress and stress 
variability; however, they can also exert an indirect effect. For example, detrimental socioeconomic circumstances, such as living in poverty, can constrain people's cognitive capacity whereby focusing on imminent and pressing problems comes at the neglect of maintenance and timely attention to issues of daily life that could avoid further stress in the future (Shah, Mullainathan, \& Shafir, 2012). People with higher trait self-control may also experience lower stress and variability in stress because they structure and plan their lives in ways that limit their exposure to potential stressors (Hofmann et al., 2012; Webb, Lindquist, Jones, Avishai, \& Sheeran, 2017). Another possible causal pathway is through more effective regulation of exogenous stress, which may reflect differences in executive functions (Diamond, 2013) and/or sleep duration and quality (Hisler et al., 2018). For example, research has shown that people's minds often wander toward unhappy or stressful thoughts (e.g., worrying about mortgage payments; Killingsworth \& Gilbert, 2010), thus a greater ability to regulate negative thought patterns might explain why people with high trait selfcontrol's report lower stress variability.

While our datasets do not allow for the direct identification of the mechanisms driving the relationship between trait self-control and stress, enriching our longitudinal model in Study 2 with additional covariates provides further insights. Accounting for differences in education and employment along the distribution of trait self-control, however, hardly affected the relationship between trait self-control and stress (see Table S9). This suggests that the observed relationship cannot be fully explained by different socioeconomic circumstances so that individual differences in regulatory abilities likely also explain why people with high trait self-control experience lower stress levels. But importantly, although we have mainly discussed how trait self-control might influence stress, we note that the link between trait self-control and stress is undoubtedly bidirectional with stress exerting equally important influences on both the development of selfcontrol as well as people's ability to use self-control (e.g., Arnsten, 2009; Blair, 2010; Hackman \& Farah, 2009). From this perspective, our results may reflect an interplay between self-control and 
stress that could lead to a prolonged negative stress spiral whereby stress weakens executive functioning and self-control which, in turn, makes stress regulation even harder.

The present results align with the ample research highlighting the numerous positive outcomes associated with trait self-control (e.g., De Ridder et al., 2012; Hofmann et al., 2014; Wiese et al., 2018). In fact, some have argued that high trait self-control is the epitome of "the good life." Nevertheless, and as alluded to above, the core mechanisms of trait self-control responsible for producing the positive outcomes remain unsettled. Tangney et al. (2004) originally defined trait self-control as the ability to override, change, or inhibit inner responses and impulses; however, later work has shown that other mechanisms may be equally or more representative of trait selfcontrol (e.g., De Ridder et al., 2012; Fujita, 2011; Hofmann \& Kotabe, 2012). For example, a recent study found little to no relationship between trait self-control and inhibition-related executive functioning (Saunders, Milyavskaya, Etz, Randles, \& Inzlicht, 2018; see also Duckworth \& Kern, 2011). Thus, the uncertainty around the core mechanisms of trait self-control highlight the need for further research to identify the processes by which trait self-control leads to desirable life outcomes, such as better mental health and lower stress.

Although the present two studies provide compelling support for the negative relationship between trait self-control and stress and its generalizability beyond student- and U.S.-based samples, they still have notable limitations. Because the two studies' predictions and analyses were not preregistered, the present evidence cannot be considered confirmatory of the relationship's generalizability. The sample in Study 1 was, despite its diversity, not representative of the populations in Germany, Poland, Sweden, or the United States. Study 2's sample had an overrepresentation of women and with a stronger educational background and left-leaning political views than the U.K. average. Consequently, we cannot claim that the negative relationship between trait self-control and stress fully generalize to the five sampled countries. The five sampled countries were also Western, and thus our research does not break with the dominance of Western 
samples in psychological research (Rad et al., 2018). We hope future research will investigate the generalizability of the examined relationships in non-Western locations.

\section{Conclusion}

Previous research has found a negative relationship between trait self-control and stress, but this relationship has so far mostly been examined in student samples. Over two large studies across five countries and using different methods, we found strong supportive evidence for trait self-control's negative relationship with stress as indicated by lower levels of stress (mean and variability). This suggests that the negative relationship between trait self-control and stress generalizes beyond students living in the United States to other population segments and countries. 


\section{References}

Arnett, J. J. (2008). The neglected 95\%: Why American psychology needs to become less American. American Psychologist, 63, 602-614.

Arnsten, A. F. (2009). Stress signalling pathways that impair prefrontal cortex structure and function. Nature Reviews Neuroscience, 10, 410-422.

Baldwin, C. L., Finley, A. J., Garrison, K. E., Crowell, A. L., \& Schmeichel, B. J. (2018). Higher trait self-control is associated with less intense visceral states. Self and Identity, 1-13.

Blair, C. (2010). Stress and the development of self-regulation in context. Child Development Perspectives, 4, 181-188.

Blair, C., \& Ursache, A. (2011). A bidirectional model of executive functions and self-regulation. In K. D. Vohs \& R. F. Baumeister (Eds.), Handbook of self-regulation: Research, Theory, and Applications (2nd ed., pp. 300-320). New York, NY: Guilford Press.

Boals, A., vanDellen, M. R., \& Banks, J. B. (2011). The relationship between self-control and health: The mediating effect of avoidant coping. Psychology \& Health, 26, 1049-1062.

Bowlin, S. L., \& Baer, R. A. (2012). Relationships between mindfulness, self-control, and psychological functioning. Personality and Individual Differences, 52, 411-415.

De Ridder, D., Kroese, F., \& Gillebaart, M. (2018). Whatever happened to self-control? A proposal for integrating notions from trait self-control studies into state self-control research. Motivation Science, 4, 39-49.

De Ridder, D., Lensvelt-Mulders, G., Finkenauer, C., Stok, F. M., \& Baumeister, R. F. (2012). Taking stock of self-control: a meta-analysis of how trait self-control relates to a wide range of behaviors. Personality and Social Psychology Review, 16, 76-99.

Diamond, A. (2013). Executive functions. Annual Review of Psychology, 64, 135-168.

Duckworth, A. L., \& Kern, M. L. (2011). A meta-analysis of the convergent validity of self-control measures. Journal of Research in Personality, 45, 259-268. 
Enders, C. K., \& Tofighi, D. (2007). Centering predictor variables in cross-sectional multilevel models: A new look at an old issue. Psychological Methods, 12, 121-138.

Farah, M. J. (2017). The neuroscience of socioeconomic status: Correlates, causes, and consequences. Neuron, 96, 56-71.

Fujita, K. (2011). On conceptualizing self-control as more than the effortful inhibition of impulses. Personality and Social Psychology Review, 15, 352-366.

Galla, B. M., \& Duckworth, A. L. (2015). More than resisting temptation: Beneficial habits mediate the relationship between self-control and positive life outcomes. Journal of Personality and Social Psychology, 109, 508-525.

Gwozdz, W., Nielsen, K. S., \& Müller, T. (2017). An environmental perspective on clothing consumption: consumer segments and their behavioral patterns. Sustainability, 9, 762.

Hackman, D. A., \& Farah, M. J. (2009). Socioeconomic status and the developing brain. Trends in Cognitive Sciences, 13, 65-73.

Hennecke, M., Czikmantori, T., \& Brandstätter, V. (2019). Doing Despite Disliking: Selfregulatory Strategies in Everyday Aversive Activities. European Journal of Personality, 33, 104-128.

Henrich, J., Heine, S. J., \& Norenzayan, A. (2010). The weirdest people in the world?. Behavioral and Brain Sciences, 33, 61-83.

Hisler, G. C., Krizan, Z., \& DeHart, T. (2019). Does Stress Explain the Effect of Sleep on SelfControl Difficulties? A Month-Long Daily Diary Study. Personality and Social Psychology Bulletin, 45, 864-877.

Hofmann, W., Baumeister, R. F., Förster, G., \& Vohs, K. D. (2012). Everyday temptations: an experience sampling study of desire, conflict, and self-control. Journal of Personality and Social Psychology, 102, 1318-1335. 
Hofmann, W., \& Kotabe, H. (2012). A general model of preventive and interventive selfcontrol. Social and Personality Psychology Compass, 6, 707-722.

Hofmann, W., Luhmann, M., Fischer, R. R., Vohs, K. D., \& Baumeister, R. F. (2014). Yes, but are they happy? Effects of trait self-control on affective well-being and life satisfaction. Journal of Personality, 82, 265-277.

Hofmann, W., Schmeichel, B. J., \& Baddeley, A. D. (2012). Executive functions and selfregulation. Trends in Cognitive Sciences, 16, 174-180.

Joanes, T. (2019). Personal norms in a globalized world: Norm-activation processes and reduced clothing consumption. Journal of Cleaner Production, 212, 941-949.

Killingsworth, M. A., \& Gilbert, D. T. (2010). A wandering mind is an unhappy mind. Science, 330, 932-932.

Lovibond, P. F., \& Lovibond, S. H. (1995). The structure of negative emotional states: Comparison of the Depression Anxiety Stress Scales (DASS) with the Beck Depression and Anxiety Inventories. Behaviour Research and Therapy, 33, 335-343.

Mischel, W., Shoda, Y., \& Rodriguez, M. I. (1989). Delay of gratification in children. Science, 244, 933-938.

Milyavskaya, M., Berkman, E. T., \& De Ridder, D. T. (2019). The many faces of self-control: Tacit assumptions and recommendations to deal with them. Motivation Science, 5, 79-85.

Moffitt, T. E., Arseneault, L., Belsky, D., Dickson, N., Hancox, R. J., Harrington, H., ... \& Sears, M. R. (2011). A gradient of childhood self-control predicts health, wealth, and public safety. Proceedings of the National Academy of Sciences, 108, 2693-2698.

Nielsen, K. S., \& Bauer, J. M. (2019). The Merits of Goal Support as a Self-Control Strategy. Social Psychological and Personality Science, 10, 671-680. 
Nielsen, K. S., Gwozdz, W., \& De Ridder, D. (2019). Unravelling the relationship between trait self-control and subjective well-being: The mediating role of four self-control strategies, Frontiers in Psychology, 10, 706.

Pickett, K. E., \& Wilkinson, R. G. (2010). Inequality: an underacknowledged source of mental illness and distress. The British Journal of Psychiatry, 197, 426-428.

Primo, D. M., Jacobsmeier, M. L., \& Milyo, J. (2007). Estimating the impact of state policies and institutions with mixed-level data. State Politics \& Policy Quarterly, 7, 446-459.

Rad, M. S., Martingano, A. J., \& Ginges, J. (2018). Toward a psychology of Homo sapiens: Making psychological science more representative of the human population. Proceedings of the National Academy of Sciences, 115, 11401-11405.

Rogers, W. H. (1993). Regression standard errors in clustered samples. Stata Technical Bulletin, 13, $19-23$.

Sapolsky, R. M. (1994). Why zebras don't get ulcers: A guide to stress, stress-related diseases, and coping. New York: Freeman.

Saunders, B., Milyavskaya, M., Etz, A., Randles, D., \& Inzlicht, M. (2018). Reported self-control is not meaningfully associated with inhibition-related executive function: A Bayesian analysis. Collabra: Psychology, 4, 39.

Shah, A. K., Mullainathan, S., \& Shafir, E. (2012). Some consequences of having too little. Science, $338,682-685$.

Simons, D. J., Shoda, Y., \& Lindsay, D. S. (2017). Constraints on generality (COG): A proposed addition to all empirical papers. Perspectives on Psychological Science, 12, 1123-1128.

Starcke, K., \& Brand, M. (2012). Decision making under stress: a selective review. Neuroscience \& Biobehavioral Reviews, 36, 1228-1248. 
Tangney, J. P., Baumeister, R. F., \& Boone, A. L. (2004). High self-control predicts good adjustment, less pathology, better grades, and interpersonal success. Journal of Personality, 72, 271-324.

vanDellen, M. R., Shah, J. Y., Leander, N. P., Delose, J. E., \& Bornstein, J. X. (2015). In good company: Managing interpersonal resources that support self-regulation. Personality and Social Psychology Bulletin, 41, 869-882.

Webb, T. L., Lindquist, K. A., Jones, K., Avishai, A., \& Sheeran, P. (2018). Situation selection is a particularly effective emotion regulation strategy for people who need help regulating their emotions. Cognition and Emotion, 32, 231-248.

Wiese, C. W., Tay, L., Duckworth, A. L., D’Mello, S., Kuykendall, L., Hofmann, W., ... \& Vohs, K. D. (2018). Too much of a good thing? Exploring the inverted-u relationship between selfcontrol and happiness. Journal of Personality, 86, 380-396.

Yang, L., Zhao, Y., Wang, Y., Liu, L., Zhang, X., Li, B., \& Cui, R. (2015). The effects of psychological stress on depression. Current Neuropharmacology, 13, 494-504. 